\title{
VOICE SERVICES FOR PSTN AND IP NETWORKS
}

\author{
Qi Guan
}

SIEMENS AG Austria

Siemensstraße 88-92, A-1210

Vienna, Austria

Key words: Voice over IP, VoIP, Services, PSTN

Abstract: This paper presents an architecture for Voice over IP services between PSTN and ISPs. The architecture is vendor independent or its vendor dependence is minimal. That means it is easily applicable for ISP's and LEC's. With this system the called party is called with his original PSTN phone number. The call is also roamable so that the called party can be reached independent of his location, of VoIP terminal or even of his ISP's. The administration of these services is configurable either by the traditional way or on the web. Thus, all these can increase the call completion rate. This benefits the IP users from not missing any important telephony calls during an Internet session. It benefits the PSTN carriers to generate revenues from both telephony calls and Internet on-line access usage at the same time. It benefits the ISPs from providing the value-added service to increase their profitability.

\section{INTRODUCTION}

On-line access becomes more and more popular in everyday life. Residential Internet subscribers access mostly the Internet by dialing into the Public Switched Telephone Network (PSTN). A study, which published in 1997 [1], indicates that the Internet users have different behavior than usual PSTN users. The traffic generated by these Internet users is also vastly different from the traffic traditionally observed on the PSTN. It results that the local exchange carriers (LEC) are facing new challenges in adapting their networks. The Internet Service Providers (ISP), which satisfy the growing 
mass market of on-line access, are facing the great challenges to become profitable since the number of ISPs continue to proliferate.

To grantee successful Internet access, ISPs must provide enough lines. While the variation of number of Internet access is large, peak load of ISPs usually only happens in the evening. This means most of the time the line side equipment (modem banks) of ISP's are not utilized efficiently.

The Internet access by dial-up lines has significant impacts on the PSTN traffic. It increases the number of busy signals per line. Even during daytime, the traditional voice busy-hours, the increase Internet traffic is considerable. This requires the LECs to rearrange of subscriber-line in concentration-stages, to increase the capacity of trunk equipment, even to purchase additional ones. On the other hand, busy terminating lines reduce the revenue of the operator, since he has to provide expensive call setup resources that do not end in completed calls.

Both local exchange carriers (LEC) and Internet Service Providers (ISP) have recognized that it is necessary to offer value-added services. The Internet telephony or more precisely Voice over IP (VoIP) is one of the new exciting opportunities for both LECs and ISPs. This new service helps LECs to hold its existing end-users, to attract new customers, to increase the telephone call completion rate, to improved the efficiency of the PSTN. For ISPs this will improve their marketing position and profitability by serving these features.

In this paper, we present a new VoIP architecture to integrate Voice over IP services between LEC of PSTN and ISPs. With this architecture more calls can be completed even while the user is in an Internet session. Call completion is an important aspect of the service provided by telecommunication operators. Calls terminate with busy or no-answer state, consuming network resources. Resolution with combination of call forwarding and Internet Call Completion contributes to greater call completion which lowers operating expense and provides better value to both the consumer and service provider. This architecture is for LEC minimal vendor dependent. It provides PSTN supplementary services like call forwarding etc., for both PSTN user and IP user. 


\section{PSTN/INTERNET INTERWORKING}

The PSTN/Internet interworking is one of the most rapidly developing fields in the telecommunication industry. It allows Internet user (or IP user) to dial up from PSTN terminal to the Internet world. With the Voice over IP (VoIP), it becomes possible to make calls from/to PSTN user while an Internet session is being engaged.

At present, a well known standard suite for VoIP is ITU H.323 protocol [2]. In H.323 four main components (called entities) are defined: Terminals, Gateways, Multipoints Control Units and Gatekeepers. An H.323 Gateway (GW) is the voice or multimedia internetworking component for connecting IP packet-based network and PSTN. An H.323 Gatekeeper (GK) is located on the Internet that provides address translation and controls access to data network for H.323 Terminals, Gateways etc. The gatekeeper may also provide other services to the Terminals, gateways and MCUs such as bandwidth management and locating Gateways.

There is another protocol to handle VoIP, this is the Session Initiation Protocol (SIP)[3] defined by IETF.

A IP user in an Internet session that uses a VoIP terminal can dial an E.164 (a usual telephone number system) code to call a PSTN user. This number in most cases must be dialed including the international code and area code. The international code or area code is needed because that the GK or SIP Server is like to build a VoIP call connection to the nearest gateway from called PSTN user.

There are many discussions for a PSTN user to call a IP user.

ETSI-TIPHON and ITU are currently investigating to assign an International-Service-Code (a.k.a. Country-Code) for IP-Telephony. This new E.164 code than could be dialed by any PSTN phone to reach a IP terminal, or dialing a gateway and the IP-address of the terminal in decimal $4 * 3$ digit notation. Dialing the gateway and a randomly assigned number is also required by some implementations.

Yet another way is that a PSTN user dial the number of the GW/GK as prefix plus the calling code of Internet user which connected to the telephone line. To become reachable, the IP user will have to first register himself to certain servers, like Gatekeeper (GK) and Location Server (LS), to indicate that he is in an Internet session and the VoIP program is running in his 
computer (possible in a background mode). When a PSTN user dials an IP user, the GK will look up the LS for the status of the IP user. If the IP user is currently engaging an Internet session, the GK will establish the connection to PC and GW sends the converted voice packets to computer. The VoIP program is activated.

The drawbacks of the methods mentioned above are obviously for both caller and called party:

- The caller doesn't know whether the called party is in the Internet session. A caller may at first dial directly to the called party per PSTN. If it is busy, the caller may dial to GW/GK. If called party is in Internet session and has been registered in GK, the call connection can be established. If the called party is not in Internet session, the call to GK/GW will also fail. Thus increases the busy traffic.

- The called party must make known his original telephone calling code and the calling code of GW/GK.

- The calling code of GW/GK usually depends on ISP (Internet Server Provider). If the called party has changed his ISP, the related GW/GK code must also be changed.

\section{A NEW VOIP ARCHITECTURE}

In this section we present a new architecture of VoIP services for interworking between PSTN LEC's and ISPs. The advantages of the system are

- The called party is being reached with his original PSTN calling code (a.k.a. phone number). The working state of called party, the ISP, and the GW/GK is transparent for caller. Neither the called status, nor the GW/GK number is a concern of caller.

- The call is roamable, which means the called party can be called, under a single, location-independent address, while the user may use different VoIP terminals on different machines in different locations, even with different ISPs.

- The interwoking implementation between PSTN and ISP is PSTN vendor independent. 
- The administration of services on PSTN side is configurable. It provides a means by either the traditional way or the web-bases application.

- The services are available for either caller or called party, either PSTN user or IP-users. The caller is not aware of whether the called party is in a PSTN or in an Internet session.

- It provides usual PSTN supplementary services like callforwarding (CFD) for both PSTN and IP-users. Other PSTN supplementary services like absent subscriber services may also be provided for IP-users.

\subsection{Overview}

Figure 1 shows the overview of the architecture of integrated VoIP services for PSTN and ISPs. The components are:

- Management Service (MS) and RADIUS server;

- Presence Service (PS) and Forwarding Service (FS);

- VoIP gateway;

- Location-server/H.323 Gatekeeper (GK) or the Session Initiation Protocol (SIP) server;

- Internet Subscriber Controlled Input via Internet (ISCI) Service;

- Switched Communication Network (SCN). As option, PSTN switches in SCN may have connection to Intelligent Network (IN);

- ISPs.

The Management Service (MS) is responsible for Internet user administration such as configuration and management, billing, user access right. In the Management Service a RADIUS client may be started in order to exchange information with RADIUS server.

RADIUS (Remote Authentication Dial In User Service) server is IETF RFC 2138 and RFC 2139 compatible. It provides functions as security, authorization and accounting. It allows for authentication (verification of user name and password) as well as configuration information (user profile), describing the type of service subscribed by the user. 
Presence Service (PS) stored the presence information of a Internet user. it serves a mean for Location Server, Gatekeeper, Forwarding Service to find, to retrieve and to be notificated of any changing the presence information.

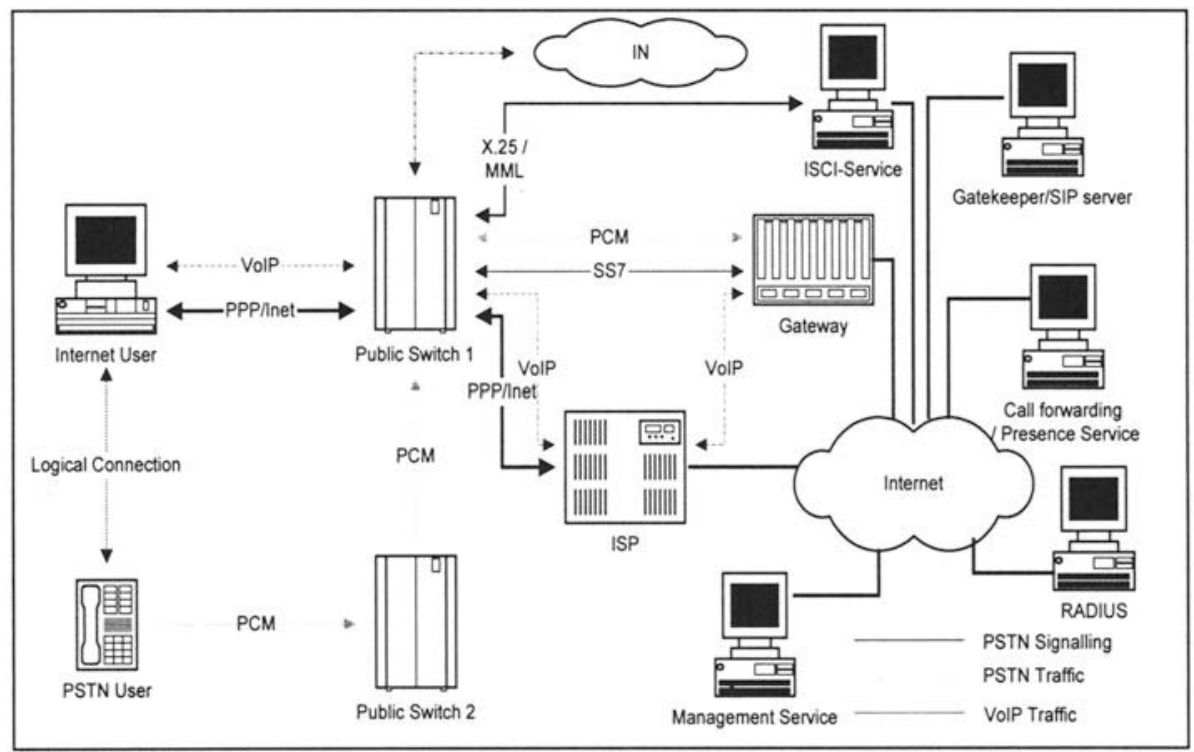

Figure 1. The architecture

Forwarding-Service (FS) is a storage for any kind of call transfer destinations for a given Internet user.

Subscriber Controlled Input via Internet (ISCI) Service provides a way for the Internet user to configure its normal PSTN telephony subscriber services through a graphical user interface by starting a WWW-application.

The PSTN switches in SCN may have an optional connection to the Intelligent Network (IN).

For convenience, the discussions below are based on H.323 standard.

\subsection{User Registration}

To use the VoIP services, an Internet user must register himself to a Gatekeeper or SIP Server through the Management Service to indicate that he is in an Internet session and the VoIP terminal is running in his computer. 
This registration is done when a user logs in to the Internet. The Management Service should verify whether the user is allowed to access this VoIP services. Therefore, the Management Service calls RADIUS to check the authentication of the user. If the user has the right to use the VoIP services, RADIUS responds a positive acknowledge. The user then makes a registration to the Gatekeeper. After the registration, the Management Service will make an IP-address available for the Internet user and store this IP-address in Presence Service(PS). The PS propagates the IP-address to Gatekeeper or SIP server and Forwarding Service (FS) if necessary.

The Internet user than can open a web browser to connect to the Subscriber Controlled Input via Internet (ISCI) Service. After authentication, the user can change his call-forwarding attributers in the subscriber database. For example, he can set his call-forwarding on busy or no reply if the subscriber access-line is analog. If the user has ISDN or broadband access, he may set call-forwarding unconditional. The destination of call-forwarding is Gateway.

Once the Internet user has registered for VoIP, configured the callforwarding service in subscriber database and Forwarding Service in Internet, he will become reachable by the caller, via PSTN or IP.

\subsection{Incoming Call Scenario}

To be reachable for either in Internet session or by PSTN phone under one single calling code, the called party needs only to set his call-forwarding service in the PSTN switch. There is no additional modifications in PSTN switch needed.

For a user using analog subscriber access-line, it is needed to set callforwarding, for example, on busy.

When a call arrived, two situations are needed to consider:

1.If the called party is in an Internet session, the call is forwarded to the VoIP Gateway. After the Gateway receives the call, it requires the Gatekeeper to check whether the called party is in an Internet session and whether the called party has the right to access VoIP services. If both criteria met, the Gatekeeper responds a positive confirmation back to the Gateway. The Gateway then looks up the Forwarding Service (FS) for the new destination of the call. If the destination is a VoIP terminal, the Gateway can then establish a connection to the caller - the PSTN user. On the other side, the Gateway converts voice 
signal into voice packages and send them to the diverted VoIP terminal (the called party's forwarding number) and vice versa. If the destination is PSTN calling code, a usual PSTN call connection will be established without Gateway participation.

2.If the called party is not in an Internet session, and phone line is busy, the call is also forwarded to the Gateway. Since there is no information of the called party in the Gatekeeper, the Gateway then should return a BUSY message back to the caller. Therefor the same state is to be delivered to the caller, the called party is busy.

\subsection{Outgoing Call from IP-user Scenario}

To make a call the Internet user must at first send a SETUP message to Gatekeeper.

The Gatekeeper than consults the Forwarding Service (FS) to see:

1. If the called party is in an Internet session and his VoIP terminal address is present the Forwarding Service (FS), the call can be directly forwarded to called party as VoIP call.

2. If there is no information of the called party, the call is forwarded to VoIP-Gateway assuming that called party is a PSTN user. Since all necessary verifications including authentication, access right etc. are completed in the registration phase, the setup process becomes more simple. Only minimal interactions between Gateway and Gatekeeper are needed. After a successful establishment of a connection to the called party - PSTN user, the Gateway converts the voice packages as PSTN signals and sends to PSTN user.

\subsection{Forwarding Service}

The Forwarding Service (FS) is used to store different types of call transfer destinations for a given Internet user / PSTN subscriber.

The call transfer destinations rely on the presence information stored in Presence Service (PS). The destinations can be either a VoIP terminal address, an e-mail address, or even another PSTN calling code.

-

If in FS the call is forwarded to a VoIP terminal, the Gateway will convert the incoming PSTN call into IP packages. If in FS the destination is Internet Call Waiting (ICW) client, the FS will acts as ICW server and communicates to the ICW Client, running on the called party's computer. 
ICW Client prompts a Dialog Box to the called party inquiring treatment for the pending call. The called party can select to send pending call to the voice mail, give it a busy signal, or give another PSTN calling code to complete the call, or even accept it by ending the current Internet connection, wait for a ring to answer the call.

For an e-mail address in the Forwarding Service, the incoming call is transferred as voice-file and is sent following the e-mail address.

For a usual PSTN calling code in Forwarding Service, the incoming call is transferred to the new PSTN call destination.

\section{W.6 Wased OA\&M service for IP-User}

Setting call-forwarding service with different options on PSTN side is one of the OA\&M (Operation, Administration and Maintenance) services provided by LEC to PSTN subscriber. A usual way is using the so called Subscriber Controlled Input(SCI) by dialing normal key-pad telephones. It is also possible to call the LEC operator. The operator sets the call-forwarding options from so-called OA\&M terminal.

Because of roaming, it is impossible for a IP-user to set his callforwarding service options from his phone. It is also difficult for a IP-user to call his LEC operator to set call-forwarding service. A better way is to allow the remote user himself to manage the call-forwarding. This can be done by the web-based OA\&M service Subscriber Controlled Input via Internet (ISCI). After authentication, a remote IP-user can query the subscriber information and set call-forwarding on busy, or unconditional or no replay accordingly. The ISCI Service sends these settings to the PSTN switch.

The interface between ISCI and PSTN switch can be either MML(ManMachine-Language) or Q3-Interface defined by ITU-T. Q3-Interface uses common management services and protocols (CMIS, CMIP) and provides a way for object oriented information exchange. Here, it is better to add an adapter between ISCI Service and OA\&M of PSTN. On one side ISCI provides a vendors independent interface, on the other side the adapter provides a vendor dependent (proprietary) control model and interface.

In this way the system minimizes the vendor dependence. For the simplest configuration, all call-forwarding is managed by key-pad telephones or by the LEC's administration operator. This is vendor independent. For the advanced configuration, all call-forwarding is managed 
by IP-user using the web-based Subscriber Controlled Input via Internet service. In both cases, we do not need to change any attributes in the subscriber database, what we change is the available attribute values.

\subsection{New Supplementary Services}

The new architecture of the VoIP for PSTN provides some new services for the VoIP user. For example,

Absent Subscriber Service,

Selective Call-forwarding, etc.

All these services are location independent.

Absent Subscriber Service is usually provided by the LEC. If, for example, the PSTN subscriber is not at home, when an incoming call arrives, LEC will turn on the voice mail, which is similar to answering machines, to take a message for the subscriber. With help of Presence Service and Subscriber Controlled Input via Internet, a remote new login IP-user can indicates the voice mail system to send the recorded voice as IP packages to computer. Thus Absent Subscriber Service together with VoIP services will prevent the user from missing messages from callers, even the user is far away from home.

Selective call-forwarding has two meanings: on PSTN switch side, it allows the Internet user to manage the call-forwarding settings by either ISCI browser or LEC operators. On the Internet side, it allows the Internet user to manage the Forwarding Service (FS). This provides a way to reroute the incoming call either to a VoIP terminal, to another PSTN phone, as voice mail, or to take the incoming call by dropping current Internet connection and waiting for a ring. The last three operations are controlled by Internet Call Waiting (ICW) Client.

\section{CONCLUSIONS}

In this paper, we presents an architecture for Voice over IP services between PSTN and ISPs. Deploying this architecture, an Internet user can be called with his/her original telephone number. It also provides a way for call roaming.

This service profits the Internet users, the LECs and ISPs. This benefits IP users from not missing any important telephony calls during an Internet session. It benefits to the PSTN carriers to generate revenues from both 
telephony calls and Internet on-line access usage at the same time. It benefits the ISPs from providing the value-added service to increase their profitability.

\section{ACKNOWLEDGMENTS}

The author would like to acknowledgment Wilhelm Wimmreuter, Josef Schmitt, Dr.Reda Reda and Polly Yu for their useful comments, suggestions and improvements to this paper.

\section{ABBREVIATIONS}

$\begin{array}{ll}\text { CMIP } & \text { Common Management Information Services Protocols } \\ \text { CMIS } & \text { Common Management Information Services } \\ \text { FS } & \text { Forwarding Service } \\ \text { GK } & \text { Gatekeeper } \\ \text { GW } & \text { Gateway } \\ \text { ICW } & \text { Internet Call Waiting } \\ \text { IN } & \text { Intelligent Network } \\ \text { ISCI } & \text { Subscriber Controlled Input via Internet } \\ \text { ISP } & \text { Internet Service Providers (ISP) } \\ \text { LEC } & \text { Local Exchange Carriers } \\ \text { IETF } & \text { The Internet Engineering Task Force } \\ \text { ITU-T } & \text { The International Telecommunication } \\ & \text { Telecommunication Standardization Sector } \\ \text { LS } & \text { Location Server } \\ \text { OA\&M } & \text { Operation, Administration and Maintenance } \\ \text { PS } & \text { Presence Service } \\ \text { PSTN } & \text { Public Switched Telephone Network } \\ \text { RADIUS } & \text { Remote Authentication Dial In User Service } \\ \text { SIP } & \text { Session Initiation Protocol } \\ \text { SCN } & \text { Switched Communication Network } \\ \text { VoIP } & \text { Voice over IP }\end{array}$

\section{REFERENCES}

[1] S. Morgan, M.Delaney: The Internet and the Local Telephone Network: Conflicts and Opportunities. XVI World Telecom Congress Proceedings. 11.1997. 
[2] ITU-T Rec. H.323v2: Packet-based multimedia communications systems. 1998.

[3] IETF Multiparty Multimedia Session Control (MMUSIC) working group and the Session Initiation Protocol (SIP). http://www.ietf.org

[4] IETF PSTN and Internet Internetworking (PINT) working group. http://www.bell-labs.com/mailing-lists/pint/

[5] Dr. U.Schoen, etc.: Convergence between Public Switching and the Internet. XVI World Telecom Congress Proceedings. 11.1997. 